\title{
Entscheidung in Dien Bien Phu. Niederlage einer Kolonialmacht im Kontext des Kalten Krieges
}

\author{
Thomas Pattinger \\ Kerngebiet: Zeitgeschichte

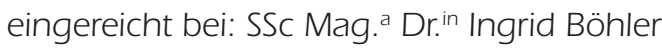 \\ eingereicht im: WS 2013/2014 \\ Rubrik: PS-Arbeit
}

\section{Abstract \\ Decision in Dien Bien Phu. Defeat of a Colonial Power in the Context of the Cold War}

Dien Bien Phu is an inconspicuous place in the middle of the undulating jungle of Vietnam next to the Laotian border. On March $13^{\text {th }} 1954$ the Viet Minh started the decisive battle against the French fortress after extensive preparations and fought the former colonial power sacrificially. France experienced its ,Stalingrad' in this battle and Vietnam gained its independence, if only briefly. It was the end of a colonial empire, but this First Indochina War also marks a hot period in context of the Cold War. The following paper deals with the circumstances that allowed the Viet Minh to win the battle.

\section{Einleitung}

„Der Kolonialmythos hatte die Kriegswirren überlebt.“" Mit diesen Worten beschreibt Dieter Brötel, Professor für Neuere Geschichte an der PH Ludwigsburg mit dem Forschungsschwerpunkt zur Geschichte des französischen Kolonialismus, treffend den Leitfaden der französischen Außenpolitik kurz nach dem Ende des Zweiten Weltkriegs. Das eigene Land stand vor den Trümmern seiner Existenz und trotzdem richtete man bald den Blick auf Indochina, das Frankreich immer stärker zu entgleiten drohte. Sorgen

1 Dieter Brötel, Schauplatz Indochina. Das Scheitern der französischen Kolonialpolitik, in: Welt und Kulturgeschichte. Epochen, Fakten, Hintergründe in 20 Bänden, Bd. 14, Hamburg 2006, S. 318-326, hier S. 320. 
bereitete vor allem ein gewisser Nguyen Van Than, ${ }^{2}$ ein Mittfünfziger mit langem Kinnbart, besser bekannt unter seinem Decknamen Ho Chi Minh. Mit ihm an der Spitze steuerten die Guerillakämpferinnen und Guerillakämpfer der Viet Minh einem geschichtsträchtigen Sieg in der Schlacht bei Dien Bien Phu entgegen, die als eine der größten Niederlagen in die Geschichte der französischen Außenpolitik eingehen sollte.

Doch welche Umstände und Taktiken erlaubten den Guerillakämpferinnen und Guerillakämpfern der Viet Minh, die Schlacht bei Dien Bien Phu für sich zu entscheiden? Dieser Frage, und jener, wie es überhaupt zu diesem Gefecht gekommen ist, soll im Folgenden genauer nachgegangen werden. Besonderes Augenmerk wird dabei auf die außenpolitische Entwicklung Frankreichs von der Zeit des Zweiten Weltkriegs an bis hin zur Friedenskonferenz von Genf im Jahr 1954 gelegt. Die daraus gewonnenen Erkenntnisse führen schließlich zu folgender These: Tiefgreifende Prozesse und Veränderungen in Bezug auf die französische Kolonialpolitik nach dem Ende des Zweiten Weltkrieges setzten schlussendlich Entwicklungen und Souveränitätsbestrebungen in Indochina in Gang, die ein Wiederherstellen des Vorkriegsstatus stark erschwerten. Das Ergebnis der Schlacht bei Dien Bien Phu ist schließlich Sinnbild für den unbändigen Willen nach Unabhängigkeit in breiten Teilen der indochinesischen Bevölkerung, die durch ungeheure Aufopferungsbereitschaft erreicht wurde.

Zur Beantwortung der gestellten Forschungsfrage und Untermauerung der zuvor genannten These wurde eine Reihe wissenschaftlicher und themenbezogener Literatur konsultiert, wobei hier besonders Ted Morgan und Dieter Brötel zu erwähnen sind. Morgan, ein Journalist und Historiker, der bereits mit dem Pulitzer-Preis ausgezeichnet wurde, besticht durch seine kritische Berichterstattung obgleich seiner französischamerikanischen Abstammung und verfasste bereits in den 1960er-Jahren Stellungnahmen zu den Entwicklungen in Südostasien. ${ }^{3}$ Brötel, seinerseits deutscher Geschichtsprofessor und Politikwissenschaftler, zeichnen seine umfangreichen Forschungen und Publikationen rund um die französische Kolonialpolitik aus. ${ }^{4}$ Besonders zu erwähnen ist im Bezug auf die verwendete Literatur außerdem das Werk "Geschichte Frankreichs seit 1945“ von Ernst Weisenfeld, dem es gelingt, stets eine Brücke zwischen den Ereignissen in Indochina und in Frankreich, aber auch den anderen Ländern, die in diesem Zusammenhang relevant erscheinen, zu schlagen und so die globale Dimension des Konfliktes im Kontext des Kalten Krieges aufzeigt.

2 Harry Thürk, Dien Bien Phu. Die Schlacht, die einen Kolonialkrieg beendete, Berlin 1994, S. 6.

3 Neben der in der Bibliographie erwähnten und in der Arbeit herangezogenen Literatur sei in diesem Zusammenhang auch noch das neueste Werk Ted Morgans erwähnt. In „Morgan, Ted, Valley of Death. The tragedy at Dien Bien Phu that led America into the Vietnam War, New York 2010 " geht Morgan stark auf die Hintergründe und politischen Ausgangslagen Frankreichs sowie Indochinas ein und erläutert umfassend, wie es schlussendlich zur Schlacht bei Dien Bien Phu kam und wie diese verlief. Stark bezieht er zudem die Rolle der Vereinigten Staaten im Kontext des Ersten Indochinakrieges mit ein und schafft ein umfangreiches und weitgreifendes Gebilde.

4 Einen kurzen und interessanten Überblick zur französischen Kolonialpolitik über Indochina hinaus gibt Brötel außerdem in einem Beitrag der Zeitschrift Francia: Brötel, Dieter, Zur französischen Imperialismusforschung und Kolonialhistorie (19./20. Jahrhundert), in: Francia - Forschungen zur Westeuropäischen Geschichte 11 (1983), Heft 1, S. 688-692. 
Aus dem gewonnenen Wissen wurde versucht, Zusammenhänge herzustellen und Schlüsse zu ziehen, soweit dies im beschränkten Rahmen einer Proseminararbeit möglich war. Die Analyse startet zeitlich mit dem Ende des Zweiten Weltkrieges und den beginnenden Spannungen zwischen Ost und West, führt über den offiziellen Beginn des Ersten Indochinakrieges hin zur entscheidenden Schlacht bei Dien Bien Phu und endet mit einem Ausblick auf die Indochinakonferenz in Genf und den getroffenen Beschlüssen sowie deren Nachwirkungen auf Frankreich und seine Kolonialpolitik.

\section{Nach dem Krieg ist vor dem Krieg}

Zwar bedeutete der Zweite Weltkrieg in Indochina und die anschließende Unabhängigkeit einstiger Kolonien eine Zäsur, „doch setzten sich im Grund Dynamiken wirtschaftlicher und sozialer Veränderungen fort, die im Kolonialismus der ersten Hälfte des 20. Jahrhunderts angelegt waren. " ${ }^{5}$ Damit ist gemeint, dass es bereits während der Kolonialzeit Bestrebungen nach Unabhängigkeit und Autonomie gab. Nach der Augustrevolution im Jahr 1945 erfolgte am 2. September desselben Jahres die Proklamation der Demokratischen Republik Vietnam (DRV) durch den kommunistisch geprägten Revolutionsführer Ho Chi Minh. Zuvor war dieser Staatsakt auf dem siebten Kominternkongress abgesegnet worden. ${ }^{6}$

Doch wer war dieser Mann, dem es gelang, mit seinen Reden die Massen zu mobilisieren und sich schließlich an die Spitze des Staates zu stellen? Ho Chi Minh, geboren 1890, wuchs in einem behüteten Umfeld auf, das ihm erlaubte, eine Schulbildung zu erhalten. Nguyen Van Than, wie er mit bürgerlichem Namen hieß, übte danach den Beruf des Lehrers aus. Er wollte jedoch mehr von der Welt erfahren und bewarb sich als Hilfskoch auf einem französischen Dampfer. Um seine Herkunft zu verschleiern, wechselte er seinen Namen und bereiste Frankreich, Spanien, Portugal, England, Irland und die afrikanischen Länder an der Mittelmeerküste. Bereits zuvor hatte er in mehreren Untergrundorganisationen gearbeitet und war daher ein vom französischen Geheimdienst gesuchter Mann. Durch den häufigen Wechsel seiner Pseudonyme versuchte er, seine Spuren zu verwischen. Zur Zeit des Ersten Weltkrieges arbeitete Ho Chi Minh als Fotograf und studierte in Frankreich und den USA. Er war mit großer Hingabe in der kommunistischen Szene Frankreichs, später auch in der Sowjetunion und in anderen Ländern tätig, ehe er nach Asien zurückkehrte und 1930 die Kommunistische Partei Indochinas gründete. ${ }^{7}$

Bereits Ende des 19. Jahrhunderts waren die fünf Territorien Laos, Kambodscha, Cochinchina (Südvietnam), Annam (Zentralvietnam) und Tongking (Nordvietnam) zu einer Indochinesischen Föderation zusammengefasst worden, die nach dem Zweiten Weltkrieg vom Hochkommissar Thierry d'Argenlieu geleitet wurde. Die Föderation war Teil der Union française, genoss jedoch große politische Autonomie. ${ }^{8}$ Während Vietnam, und hier vor allem der südliche Teil, im französischen Indochina immer stärker

5 Helmut Konrad/Monika Stromberger, Die Welt seit 1945. Einleitende Bemerkungen, in: Die Welt im 20. Jahrhundert nach 1945, hrsg. v. dens., Wien 2010, S. 13-29, hier S. 63.

6 Brötel, Schauplatz Indochina, S. 318.

7 Thürk, Dien Bien Phu, S. 6.

8 Brötel, Schauplatz Indochina, S. 321 f. 
nach Unabhängigkeit rang, strebte Frankreich indessen danach, erneut den Status der Kolonialgroßmacht zu errichten, wofür auch die gänzliche Wiedergewinnung Indochinas zentrale Bedeutung hatte. Unter Charles de Gaulle umging Frankreich die Forderungen Vietnams und arbeitete behutsam daran, die „alte Ordnung“ wieder zu errichten. ${ }^{9}$

Charles de Gaulle zog sich im Januar 1946 aus der Regierung zurück und Frankreich schien die Unabhängigkeit Vietnams anzuerkennen, bestätigte diese jedoch offiziell nicht. In einem Abkommen mit China erreichte Frankreich, dass im Norden der jungen Republik chinesische Truppen stationiert wurden. De facto wurden nach kurzer Zeit der Norden von China und der Süden von Frankreich kontrolliert, in dem d'Argenlieu am 1. Juni 1946 in Saigon die autonome Republik Cochinchina ausrief. Von dort aus plante er, auch den Norden einzunehmen und somit die Einheit der Kolonie wiederherzustellen. D'Argenlieu scheiterte jedoch mit seinem Vorhaben und war mit den Forderungen nach Einheit und Freiheit der Republik Vietnam konfrontiert. Er ließ nun Pläne schmieden, wie es den Franzosen doch noch gelingen könnte, den Norden einzunehmen. Ein Vorschlag war, "die radikalen Kräfte um den Guerillaführer Vo Nguyen Giap zu neutralisieren oder gegebenenfalls die gesamte Regierung Ho Chi Minhs zu entmachten. "10

Hinter dem Namen Vo Nguyen Giap verbirgt sich ein 1912 in Vinh geborener Politiker und Stratege aufseiten der Demokratischen Republik Vietnams. Er lehrte an der Universität Hanoi Geschichte und Geographie und trat schon früh mit Ho Chi Minh in Verbindung. Als er 1940 verhaftet werden sollte, floh er nach China. Nur zwei Jahre später kehrte er zurück und half dabei, den bewaffneten Widerstand gegen Japan zu organisieren, das zu dieser Zeit die Gebiete Indochinas besetzt hielt. Nach der Ausrufung der Republik durch Ho Chi Minh am 2. September 1945 bekleidete Giap das Amt des Innenministers. Sein strategisches Geschick verhalf inm dazu, dass er mit immer mehr militärischen Aufgaben betraut und so zum erfolgreichen Feldherrn in der Schlacht von Dien Bien Phu wurde, doch dazu später mehr. ${ }^{11}$

Die USA waren aufgrund des Wiederaufbaus in Europa auf die Stabilisierung der politischen und wirtschaftlichen Lage in Südostasien angewiesen, denn von hier bezogen sie zahlreiche Rohstofflieferungen. Aus amerikanischer Sicht sollten in Südostasien „politisch stabile, gemäßigte, prowestlich orientierte Regime“12 installiert werden. In diesem Zusammenhang unterstützten die USA immer wieder revolutionäre und antikommunistische Bewegungen, was in Moskau und Peking als Bedrohung wahrgenommen wurde. Immer stärker verlagerte sich der Konflikt des Kalten Krieges auf asiatischen Boden. Nachdem in Korea der Krieg entfesselt worden war und sich in China die Kommunistische Partei durchgesetzt hatte, wuchs die amerikanische Furcht vor einem "sowjetisch-chinesischen Block". ${ }^{13}$

9 Brötel, Schauplatz Indochina, S. 319 f.

10 Ebd., S. 323.

11 Thürk, Dien Bien Phu, S. 7.

12 Brötel, Schauplatz Indochina, S. 325.

13 Ebd., S. 324 f. 


\section{Der Kalte Krieg wird heiß}

Ein Abkommen vom 6. März 1946 zwischen Ho Chi Minh und Frankreich erkannte „,die Republik von Vietnam' als freien Staat mit Regierung, Parlament, Armee und eigenen Finanzen, aber gleichzeitig als, Teil der Indochinesischen Föderation innerhalb der Französischen Union'“14 an. Dieses Gebilde, das zu dieser Zeit nur auf dem Papier bestand, sollte nicht lange halten. Die Tinte war noch nicht getrocknet, als bereits französische Truppen mit britischer Zustimmung und Unterstützung im gesamten Land Stellung bezogen. Ho Chi Minh fühlte sich hintergangen. Der Befehlshaber der Viet Minh, General Giap, ließ am 19. Dezember 1946 in Hanoi ein französisches Gebäude stürmen und legte damit den Grundstein für den Beginn eines offiziellen Konfliktes. Einen Monat zuvor hatten Frankreichs Truppen jedoch bereits ein Massaker in Haiphong verübt, welches 6.000 Vietnamesinnen und Vietnamesen das Leben gekostet hatte. Der Sozialist Léon Blum war unterdessen Premierminister von Frankreich geworden und zog dem militärischen Konflikt eine politische Lösung vor, ohne dabei jedoch auf die ehemalige Kolonie verzichten zu wollen. ${ }^{15}$

Blum pflegte wesentlich bessere Beziehungen zu den USA als sein Vorgänger de Gaulle. Diese gewährten Frankreich großzügige Kredite und Kriegsschuldenerlässe, womit auch mehr finanzielle Mittel für den Krieg in Indochina verfügbar waren. Diese neue Finanzpolitik der USA sollte jedoch noch zum Problem Frankreichs werden, da es immer abhängiger von Washington wurde. Die französische Regierung setzte in Südvietnam den ehemaligen Kaiser von Annam, Bao Dai, als Souverän eines „unabhängigen Staates“ der Französischen Union ein, da er als Machthaber den Vorstellungen Frankreichs und der Vereinigten Staaten entsprach. Bao Dai war demnach lediglich eine Spielfigur, deren Handeln den Interessen Frankreichs entsprach. Trotzdem genoss Bai Dai ob seiner ehemaligen Position als Kaiser Ansehen und Einfluss in Teilen der Bevölkerung. Mao Zedong, Vorsitzender der Kommunistischen Partei Chinas, erkannte hingegen die Regierung Ho Chi Minhs in Vietnam an, die Anerkennung Moskaus folgte nur wenige Tage später. Die unterschiedlichen politischen Entwicklungen im Norden sowie im Süden des Landes bewirkten eine immer stärkere Teilung Vietnams. Der Kalte Krieg war heiß geworden und Vietnam wurde zum Austragungsort der Konflikte zweier Supermächte. ${ }^{16}$

In Vietnam leisteten die Guerillakämpferinnen und Guerillakämpfer rund um Ho Chi Minh und Vo Nguyen Giap erbitterten Widerstand gegen den neuerlichen französischen Einfluss. Diese kommunistisch geprägte Bewegung mit der Bezeichnung „Viet Minh“ war im Jahr 1941 in Pac Bo, einem Ort in Nordvietnam, gegründet worden. Ihre Mitglieder stammten aus den unterschiedlichsten Gesellschaftsschichten.

„Französische Kolonialisten und japanische Faschisten wurden gleichermaßen als Feinde deklariert, der Krieg als, strategisch günstige Gelegenheit' für eine politisch-militärische Befreiung begriffen. Japans Kapitulation, das durch die

14 Ernst Weisenfeld, Geschichte Frankreichs seit 1945. Von de Gaulle bis zur Gegenwart, München 1997, S. 39.

15 Ebd., S. 39 f.

16 Ebd., S. $43 \mathrm{ff}$. 
verzögerte Ankunft der Alliierten entstandene Machtvakuum und die ideologische Zersplitterung der bürgerlichen Nationalisten ermöglichten die Erfolge des Vietminh auf der Basis einer überlegenen ideologischen und organisatorischen Stärke."17

Frankreich war waffentechnisch den Truppen der Viet Minh bei weitem überlegen und konnte anfangs beinahe jedes gewünschte Territorium erobern. Die französischen Truppen kämpften jedoch fernab Europas, weshalb innen die klimatischen und geographischen Gegebenheiten kaum vertraut waren. Es gelang innen deshalb nicht, bestimmte Gebiete dauerhaft und sicher unter Kontrolle zu bringen, wodurch schon bald eine Patt-Situation erreicht war. Die französischen Truppen schafften es zwar, die Städte unter ihre Kontrolle zu bringen, doch bei der Eroberung der ländlichen Gebiete, in denen die Viet Minh lebten und dort starken Rückhalt unter großen Teilen der Bevölkerung fanden, taten sie sich ungemein schwer. ${ }^{18}$

In Washington hielt man Ho Chi Minh vorerst für eine Marionette des Kremls und betrachtete die militärischen Aktionen der Viet Minh in Indochina als eine von der Sowjetunion diktierte Offensive, die gestoppt werden müsse. Diese Meinung vertraten jedoch nicht alle amerikanischen Politikerinnen und Politiker sowie die Militärs und auch Paris stand dieser These skeptisch gegenüber. In Wirklichkeit zeigte Stalin wenig Interesse an den Vorgängen in Indochina und der Region selbst. Den Kolonialkonflikt zwischen Indochina und Frankreich bezeichnete er vielmehr als eine „ärgerliche Plage“19 und weder er noch der Kreml hegten Ambitionen, darin einzugreifen und versuchten stets, einen militärischen Ost-West-Konflikt auf vietnamesischem Boden zu vermeiden. ${ }^{20}$

Verantwortlich für die Erfolge der Viet Minh waren neben dem bereits erwähnten starken Rückhalt in breiten Teilen der vor allem ländlichen Bevölkerung auch Waffenlieferungen aus der Sowjetunion und aus China. Die beiden Staaten planten, dass im Norden Vietnams rasch eine "Transformation zum Sozialismus“21 durchgeführt werden sollte. Südvietnam mit der Hauptstadt Saigon wurde unterdessen von Bao Dai regiert, der mit einer äußerst nationalistischen Politik linke Kräfte nachhaltig eindämmte. Aus amerikanischer Sicht sollte Südvietnam ein „Bollwerk des Antikommunismus“ werden. ${ }^{22}$

\section{Die richtungsweisende Schlacht bei Dien Bien Phu}

Der Krieg in Indochina belastete Frankreich sehr und verschlang Unmengen an Ressourcen. ${ }^{23}$ Vo Nguyen Giap konnte entscheidende Erfolge in den Gefechten erzielen. Er empfand besonders große Antipathie gegenüber Frankreich, da seine Frau einst in

17 Brötel, Schauplatz Indochina, S. 318.

18 Frederik Logevall, The Indochina wars and the Cold War. 1945-1975, in: The Cambridge history of the Cold War. Volume II. Crises and Détente, hrsg. v. Melvyn P. Leffler, Cambridge 2010, S. 281-304, hier S. 283.

19 "Stalin [...] saw the conflict there as a nuisance." Logevall, The Indochina wars, S. 302.

20 Ebd.

21 Konrad, Die Welt im 20. Jh., S. 76.

22 Ebd.

23 Weisenfeld, Geschichte Frankreichs, S. 103. 
einem französischen Gefängnis gestorben war. ${ }^{24}$ Die Kampfhandlungen beschränkten sich hauptsächlich auf den Norden Vietnams und zogen sich über Jahre hin, ohne einen eindeutigen Sieger hervorzubringen. Als 1953 die Viet Minh in Laos eindrangen und dadurch Indochina aufspalteten, lehnte US-Präsident Dwight D. Eisenhower jedoch dezidiert eine militärische Intervention der USA ab. ${ }^{25}$ Eisenhower betonte allerdings bereits vor der zu diesem Zeitpunkt nicht absehbaren Schlacht bei Dien Bien Phu, dass die Vereinigten Staaten sich weiterhin in Südostasien etablieren würden. Das vermeintliche Ende der französischen Kolonialherrschaft in Indochina impliziere demnach nicht den Abbruch des amerikanischen Einflusses auf Südostasien. ${ }^{26}$

Im Frühjahr 1954 ereignete sich im Tal von Dien Bien Phu die kriegsentscheidende Schlacht. Der Ort lag eingekesselt zwischen bewachsenen Bergflanken nahe der laotischen Grenze und war von den Franzosen zu einer Festung ausgebaut worden. Sie erhofften, sich von dort aus einen entscheidenden Vorteil im Krieg verschaffen zu können, da dieser bisher jahrelang als Guerillakrieg geführt und Frankreich dadurch immer mehr in die Defensive gedrängt worden war. ${ }^{27}$ Für die Vereinigten Staaten waren die Festungen und Sicherungsanlagen Frankreichs in Dien Bien Phu der steingewordene Ausdruck einer zu defensiven Strategie, mit der Frankreich im Ersten Indochinakrieg agierte, und sie pochten auf ein aggressiveres Durchgreifen. ${ }^{28}$

Bei Dien Bien Phu planten die französischen Militärs, die Viet Minh in eine Feldschlacht zu zwingen, um die eigenen Vorteile in Kriegsführung und Technik ausnützen zu können. Unter dem Kommando des französischen Oberbefehlshabers Henri Navarre sahen sich die einstigen Kolonialherrscher anstatt des geplanten Angriffs jedoch zu einem erbitterten Verteidigungskrieg gezwungen. Der französische General Navarre hatte 60.000 Soldatinnen und Soldaten in diesem "gottverlassenen Talkessel“29 zusammengezogen. Die Versorgung dieser Stützpunkte sollte aus der Luft erfolgen. Er unterschätzte jedoch die Viet Minh gewaltig, denn diese hatten unter größten Anstrengungen Artilleriegeschütze in die Berge rund um Dien Bien Phu transportiert, mit denen sie die französischen Truppen nach einem überraschenden Angriff enorm unter Druck setzten. ${ }^{30}$ Die schweren und modernen Waffen bezogen die Viet Minh seit der kommunistischen Machtübernahme vor allem aus China. ${ }^{31}$ Giaps Truppen besetzten wichtige Verkehrswege und Flugfelder

24 Richard Cavendish, The Fall of Dien Bien Phu. May 7th, 1954, in: History Today 54 (2004), Heft 5, S. 60-61, hier S. 60.

25 Hermann Kinder/Werner Hilgemann/Manfred Hergt, dtv-Atlas Weltgeschichte. Von den Anfängen bis zur Gegenwart, München 2010, S. 515.

26 Laurent Cesari, Un malentendu transatlantique. Les États-Unis et la bataille de Diên Biên Phû, in: Guerres mondiales et conflits contemporains, 51 (2003), Nr. 211, S. 77-91, hier S. 79

27 Peter Scholl-Latour, Der Tod im Reisfeld. Dreißig Jahre Krieg in Indochina, Stuttgart 1981, S. 72

28 Nikki Cooper, Dien Bien Phu. Fifty years on, in: Modern \& Contemporary France 12 (2004), Nr. 4, S. 445457, hier S. 447

29 Scholl-Latour, Der Tod im Reisfeld, S. 72.

30 Ebd.

31 Ted Morgan, The French in Indochina. When the battle's lost and won. How the siege of Dien Bien Phu changed the modern world, in: The Economist 167 (2010), Heft 8, [http://www.economist.com/ node/15543 898], eingesehen 15.10.2013. 
und so war es den Franzosen bald nicht mehr möglich, Nachschub zu erhalten und auch ein Entkommen schien ausgeschlossen. ${ }^{32}$

Beginnend mit dem 26. April 1954 trafen sich Staats- und Regierungschefs aus Ost und West, darunter US-Außenminister John Foster Dulles, Großbritanniens Außenminister Anthony Eden, deren russischer Amtskollege Wjatscheslaw Molotow und Chinas Premierminister Zhou Enlai in Genf, um über die Zukunft Indochinas zu entscheiden. Anwesend waren auch Vertreter aus Frankreich, darunter der neu gewählte Präsident Pierre Mendès France, sowie Ho Chi Minh, der die Interessen der Viet Minh vertrat. Die Situation war aufgrund der Einkesselung von 60.000 französischen Soldatinnen und Soldaten in Dien Bien Phu angespannt. Ein Großteil der Truppen waren jedoch keine französischen Statsbürger, sondern vielmehr Söldner. ${ }^{33}$ Sie stammten hauptsächlich aus Algerien, Marokko und auch Vietnam und kämpften zusammen mit den Elite-Einheiten der französischen Fallschirmjäger. Auch vier Bataillone der Fremdenlegion, deren Offiziere zwar Franzosen, ein Großteil der unter innen dienenden Soldatinnen und Soldaten jedoch Deutsche waren, wurden im Krieg gegen die Viet Minh eingesetzt. Viele von ihnen hatten im Zweiten Weltkrieg der Deutschen Wehrmacht angehört und waren Heimkehrer von der Ostfront. ${ }^{34}$

Dien Bien Phu wurde im Zuge der Belagerung mitsamt der französischen Truppen für zwei Monate von der Außenwelt abgeschnitten. Die von den Franzosen befestigten Hügel, mit Mädchennamen getauft, gingen einer nach dem anderen verloren. Ohne Nachschub und medizinische Hilfe herrschten in den Krankenstationen der französischen Truppen katastrophale Zustände. Zahlreiche Tote beider Seiten säumten die Schlachtfelder. ${ }^{35}$ Die Stützpunkte „Gabrielle“ und „Beatrice“ mussten noch im März 1954 aufgegeben werden. Der französische Oberst Charles Piroth fühlte sich nach der Niederlage so entehrt, dass er sich mit einer Granate selbst das Leben nahm. ${ }^{36}$ Als letzten Ausweg bat der französische Außenminister Georges Bidault sogar die Vereinigten Staaten um Hilfe durch Atombomben. Der unrealistische Antrag scheiterte, obwohl Dulles zeitweise nicht abgeneigt schien. ${ }^{37}$

Während die Franzosen Stützpunkt um Stützpunkt räumen mussten, organisierten und schützten sich die Viet Minh mit ausgeklügelten Tunnelsystemen, die auch noch im Zweiten Indochinakrieg Anwendung finden sollten. Den Verlauf der Schlacht um Dien Bien Phu am 5. Mai 1954 schilderte Morgan wie folgt:

„On May 5th General Giap moved in for the kill. In three days of fighting as murderous as any of the legionnaires had seen at Stalingrad, the last trenches were overrun. Thousands of men were killed in those last days, and thousands more were taken prisoner. Four hundred were forced on a ,death march' to a

32 Cavendish, The Fall of Dien Bien Phu, S. 61.

33 Um die Kampfmoral zu stärken, wurde für die französischen Truppen in Indochina außerdem ein "mobiles Bordell“ eingerichtet, die Mädchen wurden jedoch bald als Krankenschwestern benötigt. Morgan, The French in Indochina.

34 Ebd.

35 Ebd.

36 Cavendish, The Fall of Dien Bien Phu, S. 61.

37 Scholl-Latour, Der Tod im Reisfeld, S. 73. 
prison camp 400km (250 miles) away. Later some compared the march and the camp to Dachau or Buchenwald. Others asked what else a third-world army, whose soliers received only handfuls of rice, could have done with so many more mouths to feed. "38

Nguyen Thi Ngoc Toan, ein Mediziner aufseiten der französischen Streitkräfte, war bei der Schlacht um Dien Bien Phu dabei und beschrieb die Situation, in der sich die französischen Soldatinnen und Soldaten um inn befanden, mit folgenden Worten: "The place stank, there was filth and garbage, they were stacked on top of one another, there was blood everywhere - it was like hell on earth. “39

Am 7. Mai 1954 wurde schließlich die letzte Festung genommen und die rote Flagge mit dem gelben Stern wehte als Zeichen des Sieges über dem Bunker in Dien Bien Phu. 10.000 französische Soldatinnen und Soldaten konnten festgenommen werden, doch sie waren gezeichnet von der Einkesselung, während derer es ihnen an Medikamenten und Nahrung gefehlt hatte. Nur 3.000 von ihnen sollten die Gefangenschaft überleben. ${ }^{40}$ Der Fall von Dien Bien Phu signalisierte das Ende der französischen Kolonialherrschaft in Asien. Für Frankreich war Dien Bien Phu ein Name der Scham, ein tiefer Einschnitt in der langen und ruhmreichen Geschichte französischer Militärerfolge. Auf die Meinung amerikanischer Politikerinnen und Politiker hatte die Entscheidung in Dien Bien Phu gespaltene Auswirkungen. Zwar begrüßten sie ein Ende des Kolonialismus, aber noch mehr fürchteten sie die Ausbreitung des Kommunismus in Asien. ${ }^{41}$

\section{Die Beschlüsse von Genf}

Nach fast acht Jahren blutigen Guerillakrieges gelang den Kämpferinnen und Kämpfern der Viet Minh in der Schlacht bei Dien Bien Phu am 7. Mai 1954 der entscheidende Sieg über die einstige Kolonialmacht Frankreich. Im Rahmen der Friedenskonferenz in Genf vom 26. April bis zum 21. Juli 1954 sicherte Paris den Rückzug seiner Truppen aus Indochina zu. ${ }^{42}$ In Genf wurde zudem die Teilung Vietnams in einen kommunistischen Norden und in einen kapitalistisch-autoritären Süden beschlossen. Laos und Kambodscha, ehemals Teile Indochinas, erlangten ihre Unabhängigkeit. Weiters wurden in Vietnam freie Wahlen beschlossen, das Versprechen wurde jedoch nie in die Tat umgesetzt. General Ngo Dinh Diem, der nach und nach Kaiser Bao Dai verdrängte, errichtete hingegen

38 „Am 5. Mai 1954 veranlasste General Giap einen blutigen Generalangriff. In drei Tagen des Kampfes, mörderischer, als einer der Legionäre in Stalingrad es erlebt hatte, wurden die letzten Gräben überrannt, tausende von Männern in den letzten Tagen getötet oder gefangen genommen, 400 wurden zu einem 'Todesmarsch' in ein 400 km (250 Meilen) entferntes Gefangenenlager gezwungen. Später verglichen einige den Marsch und das Lager mit Dachau oder Buchenwald. Andere fragten sich auch, was eine DritteWelt-Armee, deren Soldaten nur eine Handvoll Reis am Tag erhielten, mit so vielen Mäulern mehr, die zu stopfen waren, machen sollten." Morgan, The French in Indochina.

39 "Der Ort stank, da war Dreck und Müll, sie [Leichen, Anm.] lagen übereinandergestapelt, da war überall Blut - es war wie die Hölle auf Erden." Christopher E. Goscha, 'Hell in a Very Small Place'. Cold War and Decolonisation in the Assault on the Vietnamese Body at Dien Bien Phu, in: European Journal of East Asian Studies 9 (2010), Nr. 2, S. 201-223, hier S. 201.

40 Cavendish, The Fall of Dien Bien Phu, S. 61.

41 Morgan, The French in Indochina.

42 Brötel, Schauplatz Indochina, S. 325. 
eine Militärherrschaft, geladen mit politischen und gesellschaftlichen Spannungen, die schlussendlich zur Entfesselung eines weiteren Krieges führen sollten. ${ }^{43}$

Die geographische Teilung in Nord- und Südvietnam erfolgte entlang des 17. Breitengrades. Im Süden betrieb Ministerpräsident Diem eine vor allem von den Vereinigten Staaten diktierte Politik, die keineswegs auf Wiedervereinigung abzielte. Im Oktober des Jahres 1955 proklamierte er in Südvietnam die Republik Vietnam. Die USA nutzten diesen Stützpunkt in Asien für ihren ideologischen Kampf gegen die Ausbreitung des Kommunismus und unterstützten dabei maßgeblich das pro-westliche Regime. ${ }^{44}$

Nachdem sich Frankreich aus Indochina zurückgezogen hatte, wurde auch ein Wandel in der französischen Kolonialpolitik in Bezug auf die Kolonien in Nordafrika bemerkbar. Algerien etwa nutzte die Gunst der Stunde und es entbrannte dort am 1. November 1954 , nachdem Tunesien zuvor von Frankreich den Autonomiestatus erhalten hatte, ein regelrechter Bürger- bzw. Unabhängigkeitskrieg, der gegen Frankreich gerichtet war. ${ }^{45}$

Mario Esteban Rodríguez, Professor für Ostasienwissenschaften an der Autonomen Universität von Madrid, vertritt die Meinung, dass es hinsichtlich der Betrachtung der Kolonialpolitik Europas in Bezug auf die Länder Südostasiens keine vereinfachte Modelltheorie gibt. Er erachtet es als unzureichend, die Entwicklungen und Vorgänge in Indochina auf den Kontext des Kalten Krieges zu reduzieren, sondern verlangt nach einer vielschichtigeren Betrachtung. Als weitere wichtige Faktoren zur Analyse der Situation Indochinas zur Zeit des Ersten Indochinakrieges führt er neben dem Hintergrund des Kalten Krieges den Nationalismus in Indochina und die "Natur des Dekolonisationsprozesses"46 an, die sich auf Bestrebungen nach Eigenständigkeit in den jeweiligen Teilen Indochinas, den späteren Staaten Vietnam, Laos und Kambodscha, bezieht. ${ }^{47}$

\section{Fazit}

Ungeheurer Aufwand und Widerstandswille war seitens der Viet Minh nötig, um Frankreich schließlich in die Knie zu zwingen. Nachdem der Krieg jahrelang nur Leid und Elend, aber keine militärischen Entscheidungen gebracht hatte, entschied eine Schlacht an der laotischen Grenze über Sieg und Niederlage. Viele Faktoren beeinflussten den Ausgang des Gefechts. Einerseits war es die erfolgreiche Taktik eines durch persönliche und ideologische Antipathie angetriebenen Generals, andererseits hatte Frankreich den Gegner auch unterschätzt. Aus der Überzeugung heraus, mit militärischer Überlegenheit eine baldige Entscheidung herbeizuführen, tappten die Truppen der Grande Nation in eine Falle, aus der sie sich nicht mehr befreien sollten.

43 Hans-Ulrich Thamer, (Hrsg.), Von den Vereinten Nationen zum Kalten Krieg. Vietnam und Kambodscha (WBG Weltgeschichte. Eine globale Geschichte von den Anfängen bis ins 21. Jahrhundert 6), Darmstadt 2010, S. 93.

44 Weisenfeld, Geschichte Frankreichs, S. 103.

45 Ernst Hinrichs (Hrsg.), Kleine Geschichte Frankreichs, Bonn 2010, S. 432.

46 Rodríguez nennt in der Literatur als die drei wichtigen Faktoren „el nacionalismo, la naturaleza del proceso de descolonización y la llegada de la Guerra Fría“. Mario Esteban Rodríguez, La influencia del colonialismo occidental en las relaciones internacionales del Sudeste de Asia tras la Segunda Guerra Mundial: Ia impronta francesa en Indochina, in: Estudios de Asia y África 39 (2004), Nr. 3, S. 573-596, hier S. 595.

47 Ebd., S. 587. 
Dien Bien Phu ist viel mehr als ein kleiner, unscheinbarer Ort im Norden Vietnams. Der Name steht für den großen Sieg des indochinesischen Aufstandes gegen Bevormundung und Unterdrückung, aber auch für eine herbe Niederlage der einstigen Kolonialmacht Frankreich, die sich dadurch endgültig den Verlust des Großmachtstatus eingestehen musste. Zähneknirschend galt es, das Debakel hinzunehmen und das "Spielfeld" freizugeben, auf dem wenige Jahre später die Vereinigten Staaten und die Sowjetunion einen Kampf zweier Supermächte im Kontext politischer Disparitäten und unterschiedlicher Weltanschauungssysteme austragen sollten.

Frankreich hatte sich von dem Irrglauben leiten lassen, dass sich nach dem erstmaligen Verlust und der temporären Machtübernahme Japans, das Indochina während der französischen Abwesenheit zur Zeit des Zweiten Weltkrieges besetzt hatte, nichts geändert hatte und erlebte sein "Stalingrad“. Der Fall zeigt, welche Macht das Streben der Massen nach Unabhängigkeit, gebündelt in der Hand eines Revolutionärs wie Ho Chi Minh, entfalten kann und wie dadurch große Bevölkerungsanteile Indochinas motiviert wurden, sich mit allen ihnen zur Verfügung stehenden Mitteln gegen eine erneute Fremdherrschaft zur Wehr zu setzen. Das vietnamesische Volk hatte aus seiner Geschichte, die Jahrhunderte lang von Bevormundung und Fremdherrschaft geprägt war, gelernt, es sollte allerdings noch Jahrzehnte dauern, die Trennung des Landes zu überwinden, um schließlich endgültig als souveräner Staat zu agieren.

In großem Maße wurde der Erste Indochinakrieg auf dem Rücken der einfachen Bevölkerung ausgetragen, die am meisten darunter litt. Jedoch war stets ein breiter Rückhalt gegeben, der die Viet Minh antrieb und die unter französischer Flagge kämpfenden Soldatinnen und Soldaten fernab der Heimat zermürbte. Der Kalte Krieg war heiß geworden, die großen Machtblöcke hatten in Indochina einen Austragungsort ihrer Differenzen gefunden und die Kluft zwischen Ost und West verbreiterte sich zunehmend. Südostasien und besonders Vietnam, dessen Bewohner dies schmerzlich erfahren sollten, fungierten noch Jahrzehnte als Austragungsort des Kampfes zweier Ideologien.

\section{Literatur}

Brötel, Dieter, Schauplatz Indochina. Das Scheitern der französischen Kolonialpolitik, in: Jürgen Hotz (Hrsg.), Welt und Kulturgeschichte. Epochen, Fakten, Hintergründe in 20 Bänden, Bd. 14, Hamburg 2006, S. 318-326.

Cavendish, Richard, The Fall of Dien Bien Phu. May $7^{\text {th }}$, 1954, in: History Today 54 (2004), Heft 5, S. 60-61.

Cesari, Laurent, Un malentendu transatlantique. Les États-Unis et la bataille de Diên Biên Phû, in: Guerres mondiales et conflits contemporains, 51 (2003), Nr. 211, S. 77-91.

Cooper, Nikki, Dien Bien Phu. Fifty years on, in: Modern \& Contemporary France 12 (2004), No. 4, S 445-457.

Goscha, Christopher E., 'Hell in a Very Small Place'. Cold War and Decolonisation in the Assault on the Vietnamese Body at Dien Bien Phu, in: European Journal of East Asian Studies 9 (2010), Nr. 2, S. 201-223. 
Hinrichs, Ernst (Hrsg.), Kleine Geschichte Frankreichs, Bonn 2010.

Kinder, Hermann/Hilgemann, Werner/Hergt, Manfred, dtv-Atlas Weltgeschichte. Von den Anfängen bis zur Gegenwart, München 2010.

Konrad, Helmut/Stromberger, Monika, Die Welt seit 1945. Einleitende Bemerkungen, in: Die Welt im 20. Jahrhundert nach 1945, hrsg. v. dens., Wien 2010, S. 13-29.

Logevall, Frederik, The Indochina wars and the Cold War. 1945-1975, in: Melvyn P. Leffler, The Cambridge history of the Cold War. Volume II. Crises and Détente, Cambridge 2010 , S. 281-304.

Morgan, Ted, The French in Indochina. When the battle's lost and won. How the siege of Dien Bien Phu changed the modern world, in: The Economist 167 (2010), Heft 8 [http:// www.economist.com/node/15543898], eingesehen 15.10.2013.

Scholl-Latour, Peter, Der Tod im Reisfeld. Dreißig Jahre Krieg in Indochina, Stuttgart 1980.

Rodríguez, Mario Esteban, La influencia del colonialismo occidental en las relaciones internacionales del Sudeste de Asia tras la Segunda Guerra Mundial: la impronta francesa en Indochina, in: Estudios de Asia y África 39 (2004), Nr. 3, S. 573-596.

Thamer, Hans-Ulrich (Hrsg.), Von den Vereinten Nationen zum Kalten Krieg. Vietnam und Kambodscha (WBG Weltgeschichte. Eine globale Geschichte von den Anfängen bis ins 21. Jahrhundert 6), Darmstadt 2010, S. 93.

Thürk, Harry, Dien Bien Phu. Die Schlacht, die einen Kolonialkrieg beendete, Berlin 1994.

Weisenfeld, Ernst, Geschichte Frankreichs seit 1945. Von de Gaulle bis zur Gegenwart, München 1997.

Thomas Pattinger ist Lehramtstudent der Geschichte, Sozialkunde und Politischen Bildung, der Geographie und Wirtschaftskunde sowie der Germanistik im 6. Semester an der Universität Innsbruck. thomas.pattinger@student.uibk.ac.at

\section{Zitation dieses Beitrages}

Thomas Pattinger, Entscheidung in Dien Bien Phu. Niederlage einer Kolonialmacht im Kontext des Kalten Krieges, in: historia.scribere 7 (2015), S. 83-94, [http://historia. scribere. at], 2014-2015, eingesehen 1.3.2015 (=aktuelles Datum).

(C) Creative Commons Licences 3.0 Österreich unter Wahrung der Urheberrechte der Autorlnnen. 